\title{
OBTURATION INTESTINAL OBSTRUCTION IN THE COURSE OF NECROTIZING ENTEROCOLITIS IN NEWBORN CHILDREN
}

DOI: 10.36740/WLek202104105

\author{
Olga M. Gorbatyuk', Stanislav I. Babuci ${ }^{2}$, Taras V. Martinyuk ${ }^{3}$, Konstantin T. Bertsun ${ }^{4}$ \\ ${ }^{1}$ SHUPYK NATIONAL MEDICAL ACADEMY OF POSTGRADUATE EDUCATION, KYIV, UKRAINE \\ 2INSTITUTE OF MOTHER AND CHILD, CHISINAU, REPUBLIC OF MOLDOVA \\ 3VOLYN REGIONAL TERRITORIAL MEDICAL ASSOCIATION FOR MATERNAL AND CHILD WELFARE, LUTSK, UKRAINE \\ ${ }^{4}$ VINNYTSIA REGIONAL CHILDREN'S CLINICAL HOSPITAL, VINNYTSIA, UKRAINE
}

\begin{abstract}
The aim: To analyze our own results of diagnosis and treatment of newborns with NEC and obturation intestinal obstruction in order to determine theoretically important and practically significant recommendations on this issue, to optimize preventive measures for reducing intestinal obstruction cases in NEC and improving treatment outcome among newborns.

Materials and methods: Our supervision involved 143 newborns with NEC for the period from 2006 to 2020, including 79 boys (55.24\%) and 64 girls ( $44.76 \%$ ). Gestational age was 26-42 weeks. The majority of children were premature infants and numbered 121 children (84,62\%). The assessment of clinical manifestations, progression of the disease, physical examination of newborns were used to help in diagnosing. All children underwent general clinical tests and instrumental methods of diagnosis including ultrasonography (abdominal organs and retroperitoneum, echocardiography, neurosonography) and X-ray examination (two-dimensional plain abdominal radiography, X-ray contrast study of the gastrointestinal tract). According to the survey, all patients were examined by allied health professionals.

Results: Obturation intestinal obstruction associated with NEC was diagnosed in 26 children (18.18\%). 12 children were in serious condition, representing 46.15\%. The condition of other 14 children (53.85\%) was assessed as critical one. The first signs of intestinal obstruction were observed in children with NEC at the end of the first week or at the beginning of the second week of life. Intestinal obstruction was acute in 21 children (80.77\%); 5 children (19.23\%) had relapsing course. Blood test results showed the inflammatory process and were not specific. According to the results of our observation, the dynamics of platelets is indicative as there is a correlation between the deterioration in the child's condition and the onset of symptoms of intestinal obstruction and thrombocytopenia. There was an imbalance in protein and electrolyte metabolism - a decrease in the level of albumin, indicators of $\mathrm{K}, \mathrm{Na}, \mathrm{Ca}$, and an increase of C-reactive protein by several times. Ultrasonography of the abdominal organs showed irregular intestinal pneumatization, dilated intestinal loops with stagnant intestinal contents, pendulum peristalsis, infiltrative bowel wall thickening, free fluid in the abdominal cavity. Plain radiography revealed typical manifestations of small intestinal obstruction with multiple air-fluid levels. In 15 newborns (57.69\%), conservative treatment was effective, in particular intestinal obstruction was relieved; 11 newborns (42.31\%) underwent surgery. The mortality was $36.36 \%$ ( 4 children died). Postoperative complication in the form of bowel stenosis in the area of the direct anastomosis was observed in 3 patients approximately 2 weeks after the surgery.

Conclusions: 1. NEC can lead to the development of obturation intestinal obstruction in newborns. Obturation intestinal obstruction was a form of NEC course in $18.18 \%$ of children under our observation.

2. Obturation intestinal obstruction in NEC in newborns is a reversible condition and requires conservative therapy. If symptoms of obstruction persist within 3 days after the start of conservative therapy, surgical treatment is indicated.

3. In case of obturation intestinal obstruction, it is advisable to perform resection of necrotic bowel, formation of a double enterostomy.

4. Enterostomy closure by placing T-shaped intestinal anastomosis should be performed 4 weeks after the primary surgery.

5. The use of modern techniques for early diagnosis and timely treatment of obturation intestinal obstruction in NEC can reduce mortality and the number of postoperative complications.

6. Newborns with NEC should be under the supervision of surgeons, which will help timely determine the indications for surgical intervention.
\end{abstract}

KEY WORDS: necrotizing enterocolitis, newborns, obturation intestinal obstruction,treatment

Wiad Lek. 2021;74(4):838-841

\section{INTRODUCTION}

Obturation intestinal obstruction is a partial or complete occlusion of the intestinal lumen as a result of its blockage, external compression, or the presence of pathological process in the wall, which closes the lumen of the intestinal tube. Necrotizing enterocolitis (NEC) in newborns is a multifactorial disease which occurs as a result of severe intestinal lesion due to combined effect of potentially harmful factors and is characterized by acute inflammation of the bowel wall, dissection of gas into the bowel wall as a result of invasion of intestinal gas-forming microorganisms, ischemia and possible necrosis $[1,2,3]$.

Despite numerous epidemiological studies, the true incidence of NEC among newborns is still contradictory. Thus, 
Table I. Distribution of newborns with NEC by their number and body weight

\begin{tabular}{ccc}
\hline Body weight $(\mathbf{g})$ & Number of children $(\mathbf{n})$ & Number of children (\%) \\
\hline $500-1000$ & 12 & 8.39 \\
$1001-1500$ & 46 & 32.17 \\
$1501-2000$ & 45 & 31.47 \\
$2001-2500$ & 22 & 15.38 \\
$2501-3000$ & 11 & 7.69 \\
$3001-3500$ & 4 & 2.80 \\
greater than 3500 & 3 & 2.00 \\
\hline Overall total & 143 & 100 \\
\hline
\end{tabular}

according to the National Institute of Child Health and $\mathrm{Hu}$ man Development (the USA), the incidence of NEC in 20032007 is reported to be $11 \%$ among premature infants with a body weight of less than $1500 \mathrm{~g}$ [4]. Studies of the Canadian Neonatal Network in 2003-2008 showed that the incidence of NEC was 5.1\% [5], and the Swiss research reported 3-4\% among children less than 32 weeks of gestational age [6].

Prematurity and low birth weight are traditionally considered to be important risk factors for the development of NEC and its complications. Recent studies have shown differences in bacterial colonization, microcirculatory perfusion, maturity of gastrointestinal immunity in full-term and premature infants and these differences explain the development of obturation intestinal obstruction mainly in premature infants $[7,8,9]$.

The course of NEC may vary from nonspecific symptoms of mild local bowel lesions to systemic advanced disorders with the development of multi organ failure $[10,11]$. The progression of NEC and the development of surgical stages of the disease may occur within a few hours to 8 days from the disease onset. NEC can further lead to the development of obturation intestinal obstruction and cicatrical stenosis of the intestine. The determinants of obturation intestinal obstruction in children with NEC involve intestinal hypomotility, lack of digestive enzymes, hardening of the small bowel contents, luminal obstruction of the ileum. There are three types of obturation intestinal obstruction: intraorganic, intramural (cicatricial strictures), and extraorganic, which occurs as a result of a complete or partial extrinsic blockage of the intestinal lumen, without disturbed circulation. In newborns who required surgical interventions for intestinal obstruction, systemic manifestations of NEC prevailed over local ones, and the general condition worsened from severe to very severe [12].

In recent years, there has been a trend towards an increase in the incidence of obturation intestinal obstruction associated with NEC [13]. Treatment success depends on early diagnosis of the pathology and adequate treatment policy. Today, there is no consensus on the method of creating an anastomosis in small intestinal obstruction in newborns with NEC, which determines the relevance of this problem [15].

\section{THE AIM}

The aim of the paper is to analyze our own results of diagnosis and treatment of newborns with obturation intestinal obstruction associated with NEC in order to de- termine theoretically important and practically significant recommendations on this issue, to optimize diagnostic, therapeutic, preventive measures for reducing intestinal obstruction cases in NEC and improving treatment outcome among newborns.

\section{MATERIALS AND METHODS}

Our supervision involved 143 newborns with NEC for the period from 2006 to 2020 (67 children at Volyn Regional Children's Territorial Medical Association for Maternal and Child Welfare and 76 children at Vinnytsia Regional Children's Clinical Hospital). There were 79 boys (55.24\%) and 64 girls (44.76\%) among them. Gestational age was $26-42$ weeks. The total number of patients with NEC in absolute numbers and as a percentage of body weight is shown in Table 1 .

A child with minimum body weight was $580 \mathrm{~g}$ at the age of 26 weeks; the maximum body weight among children was $3880 \mathrm{~g}$. The majority of children were premature infants and numbered 121 children $(84.62 \%)$.

The assessment of clinical manifestations, progression of the disease, physical examination of newborns (examination, abdominal palpation and auscultation) were used to help in diagnosing. All children underwent general clinical tests (clinical blood and urine analysis, blood glucose test, blood group and $\mathrm{Rh}$ factor, protein electrophoresis, blood electrolytes, studies of urea, creatinine, total bilirubin and its fractions, ALT, AST, C-reactive protein) and instrumental methods of diagnosis including ultrasonography (abdominal organs and retroperitoneum, echocardiography, neurosonography) and X-ray examination (two-dimensional plain abdominal radiography, $\mathrm{X}$-ray contrast study of the gastrointestinal tract). According to the survey, all patients were examined by allied health professionals.

The study is carried out as part of research project of the Department of Pediatric Surgery of Shupyk National Medical Academy of Postgraduate Education entitled "Development Of New Treatment Methods And Their Assessment In Congenital and Acquired Surgical Pathology In Children" (state registration number 0118U003564).

Nonparametric statistical methods of processing medical and biological research were used in the work due to the small number of the sample and the predominance of analysis of qualitative rather than quantitative features. Numerical data are presented in absolute values (n) and percentage (\%). 


\section{RESULTS}

Obturation intestinal obstruction associated with NEC was diagnosed in 26 children (18.18\%), including:

- 4 patients with a body weight of less than $1000 \mathrm{~g}$,

- 15 newborns with a body weight of 1001-1500 g,

- 5 children with a body weight of 1501-2500 g,

- 2 children with a body weight of over $2500 \mathrm{~g}$.

The main signs of obturation intestinal obstruction involved frequent regurgitation and vomiting bile or intestinal contents, increased abdominal distention, anterior abdominal wall oedema, intestinal loop contouring, crepitus in dilated intestinal loops on palpation, gas and stool retention. The first signs of intestinal obstruction were observed in children with NEC at the end of the first week or at the beginning of the second week of life. Intestinal obstruction was acute in 21 children $(80.77 \%)$; 5 children $(19.23 \%)$ had relapsing course.

Abdominal distension, asymmetry, apnea were observed during abdominal examination. Abdominal palpation revealed local or general abdominal guarding, local or general tenderness, fluid-filled intestinal loops, pathological formations in the abdominal cavity. On auscultation, peristalsis was mostly weakened or absent.

Blood test results showed the inflammatory process and were not specific. According to the results of our observation, the dynamics of platelets is indicative as there is a correlation between the deterioration in the child's condition and the onset of symptoms of intestinal obstruction and thrombocytopenia. There was an imbalance in protein and electrolyte metabolism - a decrease in the level of albumin, indicators of $\mathrm{K}, \mathrm{Na}, \mathrm{Ca}$, and an increase of $\mathrm{C}$-reactive protein by several times.

Ultrasonography of the abdominal organs showed irregular intestinal pneumatization, dilated intestinal loops with stagnant intestinal contents, pendulum peristalsis, infiltrative bowel wall thickening, free fluid in the abdominal cavity.

$\mathrm{X}$-ray examination revealed typical manifestations of small intestinal obstruction with multiple air-fluid levels; in 2 children the obstruction was in the large intestine.

Obturation intestinal obstruction in the severe course of NEC refers to emergency neonatal surgery and can be managed conservatively or require surgical intervention.

Conservative treatment is carried out within 3 days with obligatory gastrointestinal decompression. No stimulating medicamentous therapy. In 15 newborns (57.69\%), conservative treatment was effective, in particular intestinal obstruction was relieved, passage of flatus and bowel movements resumed.

The scope of conservative therapy:

1. Cessation of enteral feeding. Total intravenous nutrition

2. Gastrointestinal decompression (washing at least 3 times a day)

3. Antibiotic therapy (Metronidazole, Amikacin, the thirdand fourth-generation Cefalosporins)

4. Infusion therapy

5. Immune therapy (Biovenum Mono, Octagam)

6. Enzyme therapy and eubiotics.

Eleven (42.31\%) out of 26 patients with intestinal obstruction underwent surgery. Among newborns who underwent sur- gery, in 7 children intestinal obstruction was localized in ileum, in 2 children - in jejunum, in 2 children - in large intestine.

The scope of surgical interventions consisted of the following:

- intestinal exploration, adheziolysis in the course of surgery

- resection of necrotic bowel, formation of a double enterostomy

- after 4 weeks - enterostomy closure by placing T-shaped intestinal anastomosis (8 cases)

- enterostomy closure by placing end-to-end intestinal anastomosis (3 cases).

All surgical interventions were accompanied by adheziolysis due to the pronounced adhesive process between the intestinal loops and the peritoneum.

The T-shaped anastomosis is an end-to-side anastomosis when the end of the proximal lumen is anastomosed to the side of the distal intestinal segment directing the end of the distal intestinal segment out in the form of enterostoma to avoid from tension on anastomosis.

After the surgery, parenteral nutrition was prescribed with clinical and biochemical blood tests control. The duration of parenteral nutrition depended on the start of enteral nutrition and the change to physiological nutritional norm i. e. 10-14 days on average.

The mortality was $36.36 \%$ ( 4 children died). The main cause of death was severe sepsis with multiple organ failure in premature infants. Postoperative complication in the form of bowel stenosis in the area of the direct anastomosis was observed in 3 patients approximately 2 weeks after the surgery.

\section{DISCUSSION}

The results of our study showed that the severe condition of a newborn with NEC, with worsening clinical manifestations of the pathology, laboratory and instrumental studies and with symptoms of progressive intestinal obstruction against the background of adequate medicamentous therapy indicated the onset and development of obturative pathology. According to the three-stage classification proposed by $\mathrm{M}$. Walsh and $\mathrm{R}$. Kleigman [14], NEC with developing obturation intestinal obstruction refers to stage III of the disease, which is the stage of surgical implications development. The child's condition progressively worsened (increased intoxication, respiratory failure, edema syndrome) in the course of NEC treatment; the symptoms of intestinal obstruction aggravated within 2-3 days. 12 children were in serious condition, representing $46.15 \%$. The condition of other 14 children $(53.85 \%)$ was assessed as critical one.

Conservative treatment is carried out within 3 days with obligatory gastrointestinal decompression. Broad-spectrum antibiotics against aerobic and anaerobic bacteria are initially prescribed. After relevant microbiological studies, the course of antibiotic therapy is corrected. Nostimulatingmedicamentoustherapy. When gas and stool were returned to normal, conservative measures were continued until the child's condition was improved; and when there were no signs of stasis, minimal enteral feeding (1-2 ml of breast milk or formula per hour) was initiated. According to our information, con- 
servative treatment was effective in $57.69 \%$ of newborns with obturative NEC.

In case of conservative treatment failure within 3 days, presence and/or worsening of clinical features of the intestinal obstruction, the surgery was indicated. Surgical treatment is initiated in patients with NEC which has clinical, roentgenologic, ultrasound signs of progressive intestinal obstruction against the background of conservative therapy failure.

The T-shaped anastomosis is an end-to-side anastomosis when the end of the proximal lumen is anastomosed to the side of the distal intestinal segment directing the end of the distal intestinal segment out in the form of enterostoma to avoid from tension on anastomosis.

The creation of a T-shaped anastomosis ensures:

- adequate emptying of the proximal intestinal segment

- the ability to introduce nutrient solutions into the distal intestinal segment, bypassing anastomosis

- wide junction between the proximal and distal intestinal segments

- the possibility to eliminate the stoma by excising the intestinal segment without intervention on anastomosis.

All practicing physicians need to know and remember about the possible occurrence and development of obturation intestinal obstruction in newborns with NEC. Timely treatment will make it possible to avoid fatal cases and the development of severe complications in a great number of patients.

\section{CONCLUSIONS}

1. NEC can lead to the development of obturation intestinal obstruction in newborns. Obturation intestinal obstruction was a form of NEC course in $18.18 \%$ of children under our observation.

2. Obturation intestinal obstruction in NEC in newborns is a reversible condition and requires conservative therapy. If symptoms of obstruction persist within 3 days after the start of conservative therapy, surgical treatment is indicated.

3. In case of obturation intestinal obstruction, it is advisable to perform resection of necrotic bowel, formation of a double enterostomy.

4. Enterostomy closure by placing T-shaped intestinal anastomosis should be performed 4 weeks after the primary surgery.

5. The use of modern techniques for early diagnosis and timely treatment of obturation intestinal obstruction in NEC can reduce mortality and the number of postoperative complications.

6. Newborns with NEC should be under the supervision of surgeons, which will help timely determine the indications for surgical intervention.

\section{REFERENCES}

1. Elgin T.G., Kern S.L., McElroy S.J.Development of the Neonatal Intestinal Microbiome and its association with Necrotizing Entarocolitis. Clin.Ther. 2016:38(4): $706-15$.

2. Samuels N., van de Graaf R.A., de Jonge R.C.J., Reiss I.K.M. Risk factors for necrotizing enterocolitis in neonates: a systematic of prognostic studies. BMC Pediatr. 2017; 17(1): 105
3. Hackam D., Caplan M. Necrotizing enterocolitis: pathophysiology from a historical context. Seminars in Pediatr. Surgery. 2018; 27(1): 11 - 18.

4. Stol B.J., Hansen N.I., Bell E.F. Neonatal outcomes of extremely preterm infants from the NICHD Neonatal Research Network. Pediatrics. 2010; 126(3): $443-456$.

5. YeeW.H., Soraisham A.S., ShahV.S. et al. Incidenceand timing of presentation of necrotizing enterocolitis preterm infants. Pediatrics.2012; 129:298-304.

6. Bajwa N.M., Berner M., Worley S., Pfister R.E. Swiss Neonatal Network. Population based age stratified morbidities of premature infants in Switzerland. Swiss Med Wkly. 2011; 141: 13212.

7. Yazji I. Endothelial TLR4 activation impairs intestinal microcirculatory perfusion in necrotizing enterocolitis via eNOS -N0-nitrite signaling. Proc. Natl Acad Sci USA. 2013; 110: 9451 - 56.

8. Neu J., Pammi M. Pathogenesis of NEC: impact of an altered intestinal microbiome. Semin Perinatol. 2017; 41:29- 35.

9. Denning N.L., Prince J.M. Neonatal intestinal dysbiosis in necrotizing enterocolitis. Molecular Medicine. 2018; 24: 4.

10. Sharma R., Hudak M.L. A clinical perspective of Necrotizing Enterocolitis: past, present and future. ClinPerinatol. 2013; 40(1): $27-51$.

11. Neu J., Pammi M. Pathogenesis of NEC: impact of an altered intestinal microbiome. Semin Perinatol. 2017; 41:29 - 35.

12. GephartS.M., Fleiner M., Kijewski A. The connection between abdominal signs and necrotizing enterocolitis in infants 501 to $1500 \mathrm{~g}$. Adv. Neonatal Care. 2017; 17(1): $53-64$.

13. Estaueb A.A., Mostafa M.M., Ibrahim N.H. The role of surgery in management of necrotizing enterocolitis. Int. J. Surg. 2010; 8(6): $458-461$.

14. Walsh M., Kleigman R. Necrotizing enterocolitis: treatment based on staging criteria. Ped. Am. 1986; 33: 179 - 201.

15. Bairov V.G., Amidkhonova S.A., Shchegoleva N.A. et al. Criteria for the choice of a method for the construction of anastomosis in newborns with small bowel obstruction. Ped.Surg. 2015; 19(1): 15 - 20.

\section{ORCID and contributionship:}

Olga M. Gorbatyuk: 0000-0003-3970-8797 A,B,E

Stanislav I. Babuci: 0000-0002-1153-4871 A,B,F

Taras V. Martinyuk: 0000-0002-0488-9148 B,C,F

Konstantin T. Bertsun: 0000-0002-2612-3709 B,C,D

\section{Conflict of interest:}

The Authors declare no conflict of interest.

\section{CORRESPONDING AUTHOR Olga M. Gorbatyuk \\ Shupyk National Medical Academy of Postgraduate Education \\ 9 Dorogozhitska str. 04112 Kyiv, Ukraine \\ tel: +380503820641 \\ e-mail:ol.gorbatyuk@gmail.com}

Received: 27.10 .2020

Accepted: 04.03 .2021

\footnotetext{
A - Work concept and design, B - Data collection and analysis, C - Responsibility for statistical analysis,
} D -Writing the article, $\mathbf{E}$ - Critical review, $\mathbf{F}$ - Final approval of the article 